\title{
LIMITED INFORMATION MAXIMUM LIKELIHOOD USING PRINCIPAL COMPONENTS AND ITS APPLICATION*
}

\author{
Haruo ONISHI**
}

\begin{abstract}
In cases where the number of predetermined variables exceeds the sample size or strong multicollinearity occurs among excluded predetermined variables, the limited information maximum likelihood and the least variance ratio cannot be used to estimate a simultaneous equation system. We propose a method in which some effectivelyinfluential principal components of excluded predetermined variables are employed as instrumental variables in place of the excluded predetermined variables. Then we demonstrate the estimation of a Cobb-Douglas agricutural production function with the proposed method.
\end{abstract}

\section{Introduction}

T.W. Anderson and H. Rubin $(1949,1950)$ proposed the limited information maximum likelihood (LIML) as a consistent estimation method for an equation in a complete simultaneous equation system. T.C. Koopmans and W.C. Hood (1953) proposed the least variance ratio (LVR) method which was found to lead to the same estimator as LIML. If a system is enlarged in order to deepen the analysis and/or prediction by it, the number of predetermined variables may exceed the number of available data, especially in economic research. Furthermore, strong multicollinearity may occur among excluded predetermined variables. In these situations, it is impossible to apply both LIML and LVR directly to the equation.

Since excluded predetermined variables are used as instrumental, we propose to employ the principal component analysis for the selection of some effectively-influential principal components from the excluded predetermined variables and then apply LIML by using the selected effectively-influential principal components in place of the excluded predetermined variables. Let us call this method the limited information maximum likelihood using principal components, abbreviated as LIPC hereafter. The method has been embodied in the Researcher System OEPP ${ }^{1}$.

* This paper is a revision of the paper which was originally presented in the Honolulu Conference on Computational Statistics held in Hawaii, Dec. 2-4, 1992.

** Institute of Socio-Economic Planning, University of Tsukuba, Tsukuba, Ibaraki, JAPAN.

Key Words: Limited information maximum likelihood; Least variance ratio; Principal component analysis; Cobb-Douglas production function; Researcher System OEPP

1 The Researcher System OEPP has been developed for variable selection for regression analysis and econometrics by the author. The System is written in FORTRAN 77, consists of about 50,000 lines in total and has about 400 subroutines and 100 functions. It has been used for research, education and governmental policy-making. 


\section{ONISHI}

\section{Notation and Assumptions}

We postulate that a symbol for a variable is also used as its data vector and a symbol for a set of variables is also used as its data matrix. Suppose that the following equation in a simultaneous equation system is estimated by using a set of excluded predetermined variables $\boldsymbol{Z}$ :

$$
Y=\boldsymbol{X} A+\boldsymbol{Y} B+U
$$

where $Y=$ LHS (left-hand side) endogenous variable or $Y=\left(y_{1}, y_{2}, \cdots, y_{T}\right)^{\prime} ; y_{t}=t$-th datum of $Y ; T=$ sample size; $t=1,2, \cdots, T ; \boldsymbol{X}=$ set of a constant term and $K$ included predetermined variables or $\{T \times(K+1)\}$-matrix of their data; $A, \boldsymbol{A}=\{(K+1) \times 1\}$-vectors of true and LIPC-estimated regression coefficients of $\boldsymbol{X}$, respectively; $Y_{\ell}=\ell$-th RHS (righthand side) endogenous variable or $(T \times 1)$-vector of its data for $\ell=1,2, \cdots, L ; \boldsymbol{Y}=$ set of $L$ RHS endogenous variables $Y_{\ell}$ 's or $(T \times L)$-matrix of their data, i.e., $\boldsymbol{Y}=\left(Y_{1}, Y_{2}, \cdots, Y_{L}\right)$; $B, \boldsymbol{B}=(L \times 1)$-vectors of true and LIPC-estimated regression coefficients of $\boldsymbol{Y}$, respectively; $U=$ disturbance term or $U=\left(u_{1}, u_{2}, \cdots, u_{T}\right)^{\prime} ; u_{t}=t$-th disturbance; and $Z=$ set of $M$ predetermined variables excluded from the equation (2.1) but included in some of the other equations and identities in the system or $(T \times M)$-matrix of their data.

We rearrange the equation $(2.1)$ into

$$
Y-\boldsymbol{Y} B=\boldsymbol{X} A+U
$$

in which the LHS and all RHS endogenous variables are gathered on the left-hand side while all included predetermined variables and the disturbance term are gathered on the right-hand side.

We rewrite, furthermore, the equation (2.2) into

$$
Y_{*}=\boldsymbol{X} A+U
$$

by introducing the following notation:

$$
Y_{*}=-\boldsymbol{Y}_{*} B_{*}, \quad \boldsymbol{Y}_{*}=(Y, \boldsymbol{Y}), B_{*}=\left(b_{0}, B^{\prime}\right)^{\prime}, \quad b_{0}=-1 .
$$

Furthermore, we use the following notation; $X_{0}=(T \times 1)$-vector of 1's, i.e., $X_{0}=$ $(1,1, \cdots, 1)^{\prime} ; \mu_{m}=m$-th eigenvalue of $\left(\boldsymbol{Z}-X_{0} X_{0}^{\prime} \boldsymbol{Z} / T\right)^{\prime}\left(\boldsymbol{Z}-X_{0} X_{0}^{\prime} \boldsymbol{Z} / T\right), P_{m}=m$-th principal component of $\boldsymbol{Z}$, corresponding to $\mu_{m}$ for $m=1,2, \cdots, M ; \mathbf{0}_{n}=(n \times 1)$-zerovector; $\boldsymbol{I}_{n}=(n \times n)$-identity-matrix; and $\sigma^{2}=$ unknown variance of $u_{t}$.

We assume that (i) the specification of the equation (2.1) is correct; (ii) $\boldsymbol{X}$ is nonstochastic or independent of all other disturbance terms, if stochastic; (iii) the equation is over-identifiable, i.e., $M>L>0$, (iv-1) the number of all predetermined variables in the system exceeds the sample size, i.e., $T<K+M+1$ or (iv-2) multicollinearity occurs among excluded predetermined variables, i.e., $\left|\boldsymbol{Z}^{\prime} \boldsymbol{Z}\right|=0$; and (v) $U \sim N\left(\mathbf{0}_{T}, \sigma^{2} \boldsymbol{I}_{T}\right)$.

\section{Principle and Estimation Procedure}

Let us explain LIPC by three steps. First of all, we define an effectively-influential principal component. If there exists strong multicollinearity between a principal component $P_{m}$ 
and the included predetermined variables $\boldsymbol{X}$, we cannot employ such a principal component for LIPC. We have to find influential principal components which are less correlated with $\boldsymbol{X}$.

By regressing each $P_{m}$ on $\boldsymbol{X}$, we obtain the residual vector $\check{E}_{m}$ as

$$
\check{E}_{m}=\left\{\boldsymbol{I}_{T}-\boldsymbol{X}\left(\boldsymbol{X}^{\prime} \boldsymbol{X}\right)^{-1} \boldsymbol{X}^{\prime}\right\} P_{m}
$$

and calculate the coefficient of determination $\pi_{m}$ as

$$
\pi_{m}=1-\frac{\check{E}_{m}^{\prime} \check{E}_{m}}{\left(P_{m}-p_{m} X_{0}\right)^{\prime}\left(P_{m}-p_{m} X_{0}\right)}
$$

for

$$
p_{m}=\frac{P_{m}^{\prime} X_{0}}{T} .
$$

Then we adjust eigenvalue $\mu_{m}$ by $\pi_{m}$ as follows:

$$
\lambda_{m}=\mu_{m}\left(1-\pi_{m}\right)^{1 / 2} .
$$

We renumber all adjusted eigenvalues $\lambda_{m}$ 's in such a way that

$$
\lambda_{1} \geq \lambda_{2} \geq \cdots \geq \lambda_{M}
$$

and also renumber the corresponding principal components. We call the $m$-th renumbered principal component $P_{m}$ the $m$-th effectively-influential principal component. Needless to say, even if $P_{m}$ is most influential but $P_{m}$ is perfectly regressed on $\boldsymbol{X}$, i.e., $\pi_{m}=1$, then $\lambda_{m}=0$ so that the corresponding principal component is treated as an uninfluential principal component. Thus, the first renumbered principal component $P_{1}$ is regarded as the most effectively-influential principal component, whereas the $M$-th renumbered principal component $P_{M}$ is regarded as the least effectively-influential principal component.

We define a rate of contribution as

$$
\zeta_{m}=100 \times \frac{\lambda_{m}}{\sum_{n=1}^{M} \lambda_{n}} \text { for } m=1,2, \cdots, M .
$$

First Step: Determine an appropriate matrix $\boldsymbol{P}$ which consists of the first $M^{\dagger}$ effectivelyinfluential principal components, $P_{1}, P_{2}, \cdots, P_{M^{\dagger}}$, under criteria $\phi_{1}(\%)$ and $\phi_{2}(\%)$, specified by a user, such that $M^{\dagger}=\max \left\{L, M^{1}, M^{2}\right\}$ where $M^{1}$ is the minimum integer among all $m$ 's which satisfy

$$
\sum_{n=1}^{m}\left\langle_{n} \geq \phi_{1}\right.
$$

and $M^{2}$ is the maximum integer among all $m$ 's which satisfy

$$
\iota_{m} \geq \phi_{2}
$$




\section{ONISHI}

and then make the following matrix:

$$
\boldsymbol{Q}=(\boldsymbol{X}, \boldsymbol{P})
$$

Second Step: Calculate $\boldsymbol{B}_{*}$ which minimizes the following variance ratio:

$$
\zeta=\frac{\boldsymbol{B}_{*}^{\prime} \boldsymbol{V}^{x} \boldsymbol{B}_{*}}{\boldsymbol{B}_{*}^{\prime} \boldsymbol{V}^{q} \boldsymbol{B}_{*}}
$$

where

$$
\begin{gathered}
\boldsymbol{V}^{x}=\boldsymbol{Y}_{*}^{\prime} \Omega^{x} \boldsymbol{Y}_{*} \\
\boldsymbol{V}^{q}=\boldsymbol{Y}_{*}^{\prime} \Omega^{q} \boldsymbol{Y}_{*} \\
\Omega^{x}=\boldsymbol{I}_{T}-\boldsymbol{X}\left(\boldsymbol{X}^{\prime} \boldsymbol{X}\right)^{-1} \boldsymbol{X}^{\prime} \\
\Omega^{q}=\boldsymbol{I}_{T}-\boldsymbol{Q}\left(\boldsymbol{Q}^{\prime} \boldsymbol{Q}\right)^{-1} \boldsymbol{Q}^{\prime}
\end{gathered}
$$

and then calculate

$$
\hat{Y}_{*}=-\boldsymbol{Y}_{*} \boldsymbol{B}_{*}=Y-\boldsymbol{Y} \boldsymbol{B}
$$

where $\boldsymbol{B}_{*}=\left(b_{0}, \boldsymbol{B}^{\prime}\right)^{\prime}$.

Third Step: Minimize the following with respect to A:

$$
\left(\hat{Y}_{*}-\boldsymbol{X} A\right)^{\prime}\left(\hat{Y}_{*}-\boldsymbol{X} A\right)
$$

Let us explain the Second Step in detail. Suppose that $\boldsymbol{B}_{*}$ is an LIPC-estimate of $B_{*}$. It is clear that the numerator of (3.14) is a sort of a variance obtained by regressing $Y_{*}$ on $\boldsymbol{X}$, since

$$
\boldsymbol{B}_{*}^{\prime} \boldsymbol{V}^{x} \boldsymbol{B}_{*}=\hat{Y}_{*}^{\prime} \Omega^{x} \hat{Y}_{*} .
$$

On the other hand, the denumerator of (3.14) is a sort of a variance obtained by regressing $Y_{*}$ on $\boldsymbol{Q}$, since

$$
\boldsymbol{B}_{*}^{\prime} \boldsymbol{V}^{q} \boldsymbol{B}_{*}=\hat{Y}_{*}^{\prime} \Omega^{q} \hat{Y}_{*} .
$$

To minimize the variance ratio (3.14) with respect to $\boldsymbol{B}_{*}$, we set the first derivative vector equal to $\mathbf{0}_{L+1}$ as

$$
\frac{\partial \zeta}{\partial \boldsymbol{B}_{*}}=\frac{2\left(\boldsymbol{V}^{x}-\zeta \boldsymbol{V}^{q}\right) \boldsymbol{B}_{*}}{\boldsymbol{B}_{*}^{\prime} \boldsymbol{V}^{q} \boldsymbol{B}_{*}}=\mathbf{0}_{L+1}
$$

so that we have

$$
\left(\boldsymbol{V}^{x}-\zeta \boldsymbol{V}^{q}\right) \boldsymbol{B}_{*}=\mathbf{0}_{L+1} .
$$


Limited Information Maximum Likelihood Using Principal Components and Its Application

For $\boldsymbol{B}_{*}$ of (3.24) to assume a nontrivial solution, the determinant of $\boldsymbol{V}^{\boldsymbol{x}}-\boldsymbol{\zeta} \boldsymbol{V}^{q}$ must be zero, i.e.,

$$
\left|\boldsymbol{V}^{x}-\zeta \boldsymbol{V}^{q}\right|=0 .
$$

Let $\zeta_{\ell}$ be the $\ell$-th eigenvalue of $(3.25)$ and

$$
\zeta^{*}=\min \left\{\zeta_{1}, \zeta_{2}, \cdots, \zeta_{L+1}\right\}
$$

which is the minimum among all eigenvalues $\zeta_{\ell}$ 's.

The LIPC-estimator $\boldsymbol{B}_{*}$ is obtained by solving

$$
\left(\boldsymbol{V}^{x}-\zeta^{*} \boldsymbol{V}^{q}\right) \boldsymbol{B}_{*}=\mathbf{0}_{L+1} \quad \text { with } \quad b_{0}=-1 .
$$

If $M^{\dagger}-L$ is less than $T$ but rather large compared with $T$, we can modify the LIPCestimator by following K. Morimune (1983) and have the following estimator satisfying:

$$
\left(\boldsymbol{V}^{x}-\zeta^{+} \boldsymbol{V}^{q}\right) \boldsymbol{B}_{*}=\mathbf{0}_{L+1} \quad \text { with } \quad b_{0}=-1
$$

where

$$
\zeta^{+}=\zeta^{*}-\frac{1}{T-K-M^{\dagger}-1}\left\{1+\frac{M^{\dagger}-L}{T-K-M^{\dagger}-1} \frac{1+\zeta^{*}}{\sum_{\ell=1}^{L+1}\left(\zeta^{*}-\zeta_{\ell}\right)}\right\} .
$$

The case in which we put $M^{\dagger}=L$ in (3.29) coincides with the Fuller modification (1977). The estimate $\hat{Y}$ of $Y$ is calculated as

$$
\hat{Y}=\boldsymbol{X} \boldsymbol{A}+\boldsymbol{Y} \boldsymbol{B}
$$

and the residual vector $\hat{\boldsymbol{E}}$ is obtained as

$$
\hat{\boldsymbol{E}}=Y-\hat{Y} \text {. }
$$

The variance $\sigma^{2}$ of the disturbance term $U$ is estimated by

$$
s^{2}=\frac{\hat{\boldsymbol{E}}^{\prime} \hat{\boldsymbol{E}}}{T-K-L-1} .
$$

The covariance matrix $\Theta$ of estimated regression coefficients $\boldsymbol{A}$ and $\boldsymbol{B}$, following T.C. Liu and W.J. Breen (1969), may be calculated as

$$
\Theta=s^{2}\left(\begin{array}{cc}
\boldsymbol{\Delta} & -\boldsymbol{\Lambda} \\
-\boldsymbol{\Lambda}^{\prime} & \boldsymbol{\Gamma}
\end{array}\right)
$$

where

$$
\begin{gathered}
\boldsymbol{\Gamma}=\left\{\boldsymbol{Y}^{\prime}\left(\zeta^{*} \Omega^{x}-\Omega^{q}\right) \boldsymbol{Y}\right\}^{-1} \\
\boldsymbol{\Lambda}=\boldsymbol{\Gamma} \boldsymbol{Y}^{\prime} \boldsymbol{X}\left(\boldsymbol{X}^{\prime} \boldsymbol{X}\right)^{-1} \\
\boldsymbol{\Delta}=\left(\boldsymbol{X}^{\prime} \boldsymbol{X}\right)^{-1}\left\{\boldsymbol{I}_{K+1}+\boldsymbol{X}^{\prime} \boldsymbol{Y} \boldsymbol{\Gamma} \boldsymbol{Y}^{\prime} \boldsymbol{X}\left(\boldsymbol{X}^{\prime} \boldsymbol{X}\right)^{-1}\right\}
\end{gathered}
$$




\section{ONISHI}

\section{An Application of LIPC to Agricutural Production Function}

Before demonstrating the estimation of a Cobb-Douglas agricultural production function with LIPC, let us see the properties of a Cobb-Douglas type production function. Let $Y$ =agricultural outputs; $X_{1}=$ non-physical index variable like technical progress; $Y_{\ell}=\ell$-th physical input like land, capital or labor for $\ell=1,2,3 ; U=$ disturbance term; and $e=$ base of a natural logarithm. Then a Cobb-Douglas production function may be expressed as

$$
Y=c e^{a_{1} X_{1}} Y_{1}^{b_{1}} Y_{2}^{b_{2}} Y_{3}^{b_{3}} e^{U}
$$

where $c, a_{1}, b_{1}, b_{2}, b_{3}=$ regression coefficients to be estimated.

Each $b_{\ell}$ for $\ell=1,2,3$ represents the production elasticity $\varepsilon$ of input as

$$
\varepsilon=\frac{\partial Y / Y}{\partial Y_{\ell} / Y_{\ell}}=\frac{\partial Y}{\partial Y_{\ell}} \frac{Y_{\ell}}{Y}=b_{\ell}
$$

If it is a priori known that all physical inputs increase the agricultural outputs, we should have as reasonable signs

$$
b_{1}>0, b_{2}>0 \text { and } b_{3}>0 .
$$

If $b_{\ell}<0$, then no use of the $\ell$-th physical input $Y_{\ell}$ increases the agricultural outputs $Y$. This contradicts the fact. The estimated regression coefficients must satisfy (4.39). Let us multiply all physical inputs $Y_{1}, Y_{2}, Y_{3}$ by $\xi>0$ and denote the corresponding agricultural outputs by $Y_{\xi}$. Then we have

$$
\begin{aligned}
Y_{\xi} & =c e^{a_{1} X_{1}}\left(\xi Y_{1}\right)^{b_{1}}\left(\xi Y_{2}\right)^{b_{2}}\left(\xi Y_{3}\right)^{b_{3}} e^{U} \\
& =\xi^{b_{1}+b_{2}+b_{3}} c e^{a_{1} X_{1}} Y_{1}^{b_{1}} Y_{2}^{b_{2}} Y_{3}^{b_{3}} e^{U} \\
& =\xi^{b_{1}+b_{2}+b_{3}} Y .
\end{aligned}
$$

Therefore, if $b_{1}+b_{2}+b_{3}>1$, then it is said that increasing returns to scale prevail because $Y_{\xi}>\xi Y$. If $b_{1}+b_{2}+b_{3}=1$, then it is said that constant returns to scale prevail in agricultural production because $Y_{\xi}=\xi Y$. If $b_{1}+b_{2}+b_{3}<1$, then it is said that decreasing returns to scale prevail because $Y_{\xi}<\xi Y$. However, if $b_{1}+b_{2}+b_{3}$ is much greater than 1 , e.g., 1.5, implying that agricultural production is extremely efficient, we must investigate from a realistic viewpoint whether or not such highly increasing returns to scale prevail. On the other hand, if $b_{1}+b_{2}+b_{3}$ is much less than 1 , e.g., 0.7 , then it is unrealistic because such a production is quite inefficient, implying that farmers cannot have room to expand their farm size. Analysis or prediction by an unrealistic regression equation draws a wrong conclusion. Accordingly, we expect together with (4.39)

$$
b_{1}+b_{2}+b_{3} \sim 1 \text {. }
$$

The above conditions on the signs and magnitude for regression coefficients of all physical inputs are required as natural logic or professional knowledge. Then statistical and data-analytic tests should follow to obtain a scientifically reasonable as well as statistically significant regression equation.

Taking logarithms of both sides of the equation (4.37), we have 
Limited Information Maximum Likelihood Using Principal Components and Its Application

$$
\log Y=d+a_{1} X_{1}+b_{1} \log Y_{1}+b_{2} \log Y_{2}+b_{3} \log Y_{3}+U
$$

where $d=\log c$. The equation (4.42) is estimated instead of the equation (4.37) after transforming variables $Y, Y_{1}, Y_{2}$ and $Y_{3}$ into $\log Y, \log Y_{1}, \log Y_{2}$ and $\log Y_{3}$ by a package, respectively.

We are in a position to demonstrate the estimation of a Cobb-Douglas agricutural production function in Japan with LIPC. Since LIPC is installed in the Researcher System OEPP, we follow the entry style of variables used in it. Variables are entered as follows:

$$
Y=F(X: Y: Z)
$$

We use the following notation:

$L Y=$ logarithm of agricultural outputs; $@ C=$ constant term; $T T=$ time trend as an index of technical progress; $L C A X=$ logarithm of cultivated acreage minus set-aside and damaged acreage; $L K R=$ logarithm of adjusted agricultural capital, consisting of animal, plant and machinery capital, in which machinery capital is adjusted by use rates of machines; $L L=$ logarithm of agricultural labor; $L W I Q=$ logarithm of wheat import quantity; $L R F I=\operatorname{logarithm}$ of real farm income; $L R R P P=\operatorname{logarithm}$ of real rice producer price determined by the government; $L R W R P F=\operatorname{logarithm}$ of man's real wage rate for parttime farming; $L K A=$ logarithm of animal capital (cows, steers, horses, pigs, sheep, etc.); $L K P=$ plant capital (trees of apple, cherry, orange, peach, pear, persimmon, grape, tea, etc.); $L K M=$ machinery capital (rice planters, harvesters, trucks, combines, silos, etc.); $L K M R=$ machinery capital adjusted by use rates of machines; and $L R U F P=$ logarithm of real ureal fertilizer price. Variable $L R F I(-1)$, for instance, represents a one-time-point lagged variable of (current) variable $L R F I$.

Econometric variable classifications are: $L Y=$ LHS endogenous variable; $T T=$ included predetermined variable; $L C A X, L K R, L L=$ RHS endogenous variables; and $L W I Q$, $L R F I, L R R P P, L R W R P F, L R F I(-1), L K A(-1), L K P(-1), L R U F P, \operatorname{LRRPP}(-1)$,

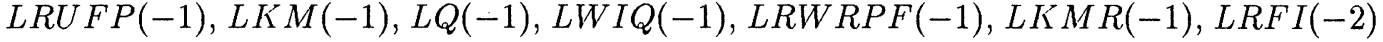
$=$ excluded predetermined variables.

We loaded the following into a FACOM M-1800/20 with a cumulative-contribu- tionrate criterion value $\phi_{1}=80(\%)$ and a marginal-contribution-rate criterion value $\phi_{2}=5(\%)$ :

$$
\begin{aligned}
L Y= & F(@ C, T T: L C A X, L K R, L L: L W I Q, L R F I, L R R P P, L R W R P F \\
& L R F I(-1), L K A(-1), L K P(-1), L R U F P, L R R P P(-1), L R U F P(-1), \\
& L K M(-1), L Q(-1), L W I Q(-1), L R W R P F(-1), L K M R(-1) \\
& L R F I(-2))
\end{aligned}
$$

where $L Y, T T, L C A X, L K R$ and $L L$ correspond to $\log Y, X_{1}, \log Y_{1}, \log Y_{2}, \log Y_{3}$ in (4.42), respectively.

The estimated regression equation is as follows: 


\section{ONISHI}

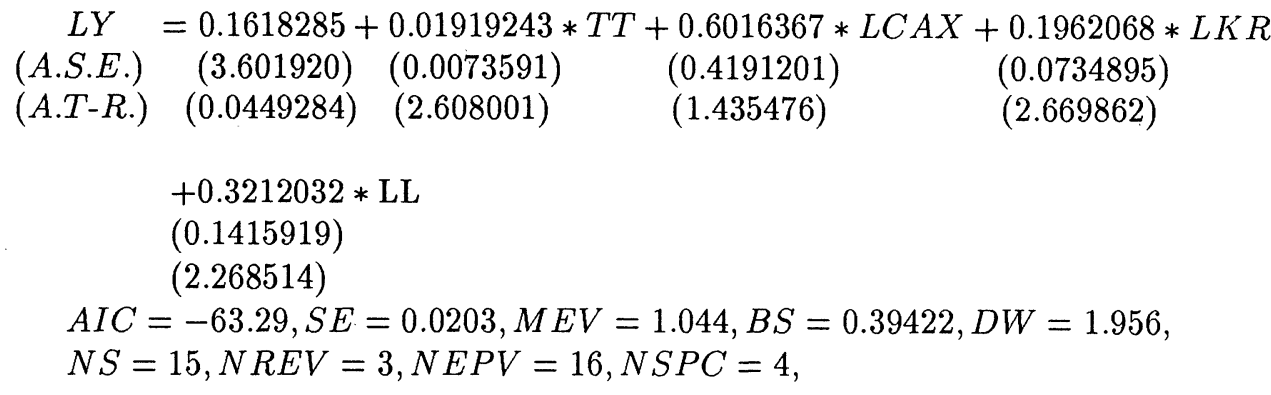

where A.S.E. =asymptotic standard errors of the estimated regression coefficients, $A . T$ $R$. =asymptotic t-ratios of the estimated regression coefficients, $A I C=$ Akaike information criterion statistic, $S E=$ asymptotic standard error of a disturbance term, $M E V=$ minimum eigenvalue, $B S=$ Basmann over-identifiability restriction statistic, $D W=$ Durbin-Watson serial correlation statistic, NS=number of samples, $N R E V=$ number of RHS endogenous variables, $N E P V=$ number of excluded predetermined variables, and $N S P C=$ number of selected effectively-influential principal components.

Let us examine the estimated regression equation (4.45). The estimated regression coefficients of $T T$ (time trend for technical progress), $L C A X$ (land), $L K R$ (capital) and $L L$ (labor) are all reasonably positive, whereas the sum of the regression coefficients of $L C A X$, $L K R$ and $L L$ is $0.6016367+0.1962068+0.3212032=1.1190467$. The degree of increasing returns to scale estimated is higher than expected but it is not too bad. On the other hand, the regression coefficients of TT, LKR and LL are asymptotically significant at a $5 \% t$-test, while that of LCAX is asymptotically significant at a $10 \% t$-test. There is no serial correlation at a $5 \%$ Durbin-Watson serial correlation test. Accordingly, the estimated agricultural production function (4.45) seems to be acceptable.

\section{Concluding Remarks}

LIML and LVR cannot be used, if the number of all predetermined variables in a simultaneous equation system exceeds the sample size or strong multicollinearity occurs among excluded predetermined variables. In these situations we can apply the principal component analysis for excluded predetermined variables, select some effectively-influential principal components for the excluded predetermined variables, and then estimate a regression equation with LIML or LVR by replacing all excluded predetermined variables with the selected principal components. We successfully demonstrated how LIPC worked for the estimation of an agricultural production function, although fairly-high increasing returns to scale were seen.

\section{REFERENCES}

Akaike, H. (1973). Information theory and an extension of the maximum likelihood principle, Proc. of 2nd inter. symp. inform. theor., B.N. Petrov and F. Csaki (eds), 178-196.

Anderson, T.W. and Rubin, H. (1949). Estimation of the parameters of a single equation in a complete system of stochastic equations, Annals of Math. Statist., 21, 570-581. 
Limited Information Maximum Likelihood Using Principal Components and Its Application

Anderson, T.W. and Rubin, H. (1950). The asymptotic properties of estimators of the parameters of a single equation in a complete system of stochastic equations, Annals of Math. Statist., 21, 570-581.

Basmann, R.L. (1960). On finite sample distributions of generalized classical linear identifiability test, J. Amer. Assoc., 55, 292, 650-659.

Durbin, J. (1975). Testing for serial correlation in systems of simultaneous regression equations, Biometrika, 44 3, 370-377.

Fuller, W.A., (1977). Some properties of of a modification of the limited information estimator, Econometrica, 45, 4, 939-953.

Hood, W.C. and Koopmans, T.C., (1953). Studies in Econometric Methods, Cowles Foundation, Yale University Press.

Liu, T.C. and Breen, W.J., (1969). The covariance matrix of the limited information estimator and the identification test, Econometrica, 37, 2, 222-227.

Morimune, K., (1983). Approximate distribution of the k-class estimators when the degree of overidentifiability is large compared to the sample size, Econometrica, 51, 3, 821841.

Onishi, H. (1993). Researcher System OEPP for Econometrics: I and II, 2nd version, OEPP Institute, Tsukuba, Ibaraki.

(Received May 1993; Revised August 1993) 